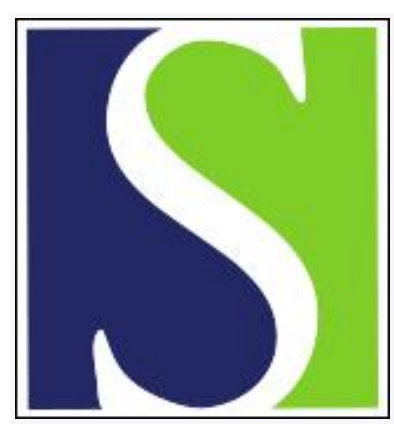

Scand J Work Environ Health 1991;17(1):1-6

https://doi.org/10.5271/sjweh.1740

Issue date: Feb 1991

Implanted medical devices in workers exposed to radio-frequency radiation.

by Hocking $\mathrm{B}$, Joyner $\mathrm{KH}$, Fleming $\mathrm{AH}$

Affiliation: Corporate Human Resources, Melbourne, Australia.

This article in PubMed: www.ncbi.nlm.nih.gov/pubmed/2047801 


\title{
Implanted medical devices in workers exposed to radio-frequency radiation
}

\author{
by Bruce Hocking, MBBS, FACOM, ${ }^{1}$ Ken $\mathrm{H}$ Joyner, $\mathrm{PhD},{ }^{2}$ Anthony $\mathrm{HJ}$ Fleming, $\mathrm{MSc}^{2}$
}

\begin{abstract}
HOCKING B, JOYNER KH, FLEMING AHJ. Implanted medical devices in workers exposed to radiofrequency radiation. Scand $J$ Work Environ Health 1991;17:1-6. This paper discusses the management of radio-frequency radiation workers who have implanted medical devices which may be adversely affected by such radiation fields. The implants include orthopedic devices, cardiac pacemakers, and cochlear implants, but exclude dental work. The effect of radio-frequency radiation on the devices may be to increase heat load and/or produce signal interference. The mechanics of interaction are outlined and protocols for managing cases are described. The implications for safety standards are discussed.
\end{abstract}

Key terms: orthopedic devices, cardiac pacemakers, cochlear implants, heat load, electromagnetic interference.

Physicians should be aware that implanted medical devices may interact in various ways with radiofrequency radiation (RFR), and this problem should be considered when they are placing or rehabilitating staff who are occupationally exposed to RFR. This review begins by noting the biophysics and occupations involved; then discusses two different mechanisms of adverse interaction, heating and electromagnetic interference; and also considers how these interactions may be managed.

The range of metallic medical devices is increasing yearly. They include orthopedic wires, plates, rods and joint replacements, cardiac valves and pacemakers, transcutaneous drug delivery systems, and cochlear implants. The Australian standard AS2772 sets exposure limits for RFR workers (figure 1), but, unlike other national and international standards, recognizes that staff with metallic implants require specific consideration (1). However, little information is available on this subject, and this review offers some guidance based on our experience.

\section{Absorption and exposure}

RFR is that portion of the electromagnetic spectrum usable for radio communications, and it is generally considered to encompass frequencies in the range of $10 \mathrm{kHz}$ to $300 \mathrm{GHZ}$. Microwave radiation is a subset of the RFR range and covers frequencies between $300 \mathrm{MHz}$ to $300 \mathrm{GHz}$.

Some of the occupational categories which may involve exposure to RFR are shown in table 1.

1 Corporate Human Resources, Melbourne, Australia.

2 Telecom Research Laboratories, Melbourne, Australia.

Reprint requests to: Dr B Hocking, Telecom Australia, 199 William Street, Melbourne, Victoria, Australia 3000.

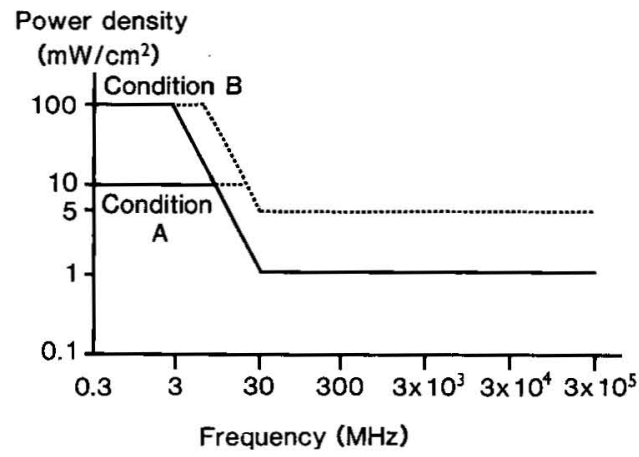

Figure 1. Occupational exposure limits for radio-frequency radiation (from AS2772). (solid lines=long-term exposure limits; $A \& B=$ possibility of shocks and burns occurring or not occurring, respectively; broken line = maximum short-term exposure level $(6 \mathrm{~min})(1)$.

Table 1. Uses of radio-frequency radiation (RFR). Various occupational categories are shown with the possible range of RFR frequencies which may be encountered.

\begin{tabular}{lc}
\hline Occupation & Frequency \\
\hline $\begin{array}{l}\text { Radiocommunications } \\
\text { Heating of wood and plastic } \\
\text { (RF welders and dryers) }\end{array}$ & $10 \mathrm{kHz}-300 \mathrm{GHz}$ \\
$\begin{array}{l}\text { Cooking (microwave ovens) } \\
\text { Heating of metals (induction } \\
\text { furnaces and some welding } \\
\text { processes) }\end{array}$ & $10 \mathrm{MHz}-100 \mathrm{MHz}$ \\
$\begin{array}{l}\text { Radar } \\
\text { Telemetry }\end{array}$ & $2.45 \mathrm{GHz}$ \\
$\begin{array}{l}\text { Navigation } \\
\begin{array}{l}\text { Medicine (eg, diathermy, cancer } \\
\text { hyperthermia treatment) }\end{array}\end{array}$ & $50 \mathrm{~Hz}-2 \mathrm{MHz}$ \\
$\begin{array}{l}\text { Analysis of chemicals and nuclei } \\
\text { (eg, nuclear magnetic resonance) }\end{array}$ & $10 \mathrm{MHz}-20 \mathrm{GHz}$ \\
\hline
\end{tabular}


The human body acts as an antenna when exposed to RFR. The energy absorption within the body during RFR exposure is a complicated function of the frequency of the RFR, the orientation of the electric-field component of the RFR relative to the long axis of the body, the size of the body or an organ relative to the wavelength of the RFR, the shape of the body or an organ, the electrical properties of the tissues, and the amount of energy contained in the RFR.

For an upright adult $1.75 \mathrm{~m}$ tall, the energy absorption averaged over the body varies with frequency as shown in figure 2 (2). It reaches a maximum at approximately $80 \mathrm{MHz}$ because the body acts as a resonant antenna when the height to wavelength ratio is approximately 0.4 . The curve of the Australian standard (figure 1) is based on protection from this absorption pattern.

The absorbed energy is lost through the movement of free ions causing an electric current (conduction loss) and through molecular rotation (dielectric loss). Thus RFR propagating through a biological medium interacts with it, and energy transfer occurs. This phenomenon results in attenuation of the RFR and an increase in the kinetic energy of the molecules of the medium (ie, in heating). The degree of attenuation of the RFR depends on the dielectric properties of the medium, and these properties also change with the frequency of the incident field (3).

Metallic implants act as antennas when exposed to RFR and thereby enhance the local electric field. As the absorbed energy is directly proportional to the square of the in-situ electric field, metallic implants enhance the absorption of energy in the vicinity of the implant. The effect of this enhanced energy absorption is an increase in the local heat load.

With some devices (eg, pacemakers and cochlear implants) the absorbed energy may also affect the oper-

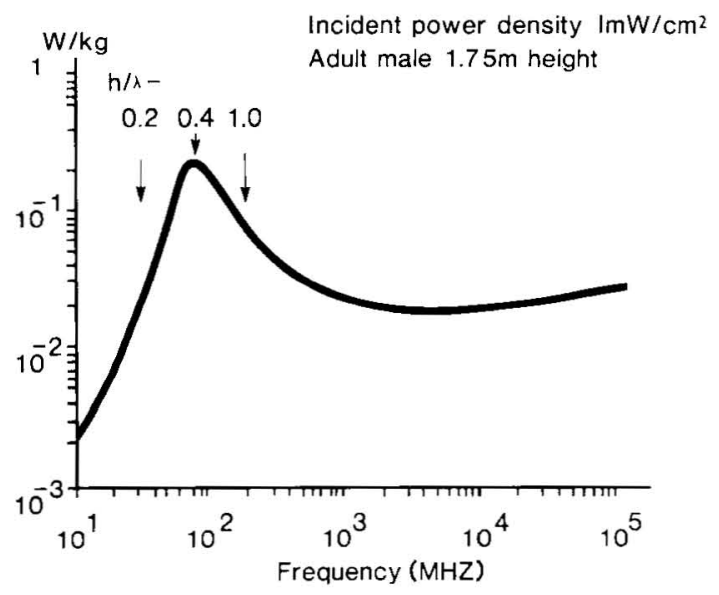

Figure 2. Calculated energy absorption averaged over the body mass $(W / k g)$ versus frequency. Maximum energy absorption occurs when the ratio of the height $(h)$ of the person to the wave length $(\lambda)$ of the radiation equals 0.4 , at which point resonance occurs. As the ratio of height to wavelength changes, energy absorption decreases rapidly (according to reference 2 ). ation of the device. Interference may enter a cardiac pacemaker either directly through the pulse generator or indirectly through an antenna which may be formed by the lead system (4). Interference with cochlear implants occurs through the direct reception of radio signals (5).

\section{Heating}

Metallic implants exposed to RFR increase local energy absorption by enhancing the local electric field; if they are thin enough, the implants may become heated, possibly adversely affecting tissue. In general, the effect is maximal when the implant is resonant, and resonance depends on the dimensions of the implant relative to the wavelength of the RFR in the tissue. The degree of heating can be calculated with the methods given in the appendix and described elsewhere $(6,7)$. Orthopedic devices are of obvious concern but other devices need consideration. There has been a case report of a skin burn in a person wearing a transdermal drug delivery system. The person was exposed to a leaky microwave oven (8). New devices, such as implanted insulin infusion systems, will also require assessment (9).

It should be noted that dental work poses a negligible hazard because the mouth is an efficient cooling device and the teeth are heat resistant. Therefore concern about heating does not apply to dental work.

The following approach to implanted devices is used in Telecom Australia (7). Details on the implant are obtained, including its configuration and anatomic location, a radiograph taken at the time of the operation being useful. Details of the potential RFR exposure are obtained. They must include the frequencies, the field strengths, and the durations of exposure. The likely heating effect of these exposures on the implant is then calculated according to the methods described by Fleming \& Joyner (6), the worst-case estimates being based on the upper limits of exposure in the Australian standard (figure 1). The extent of heating is then assessed with regard to the hazard to adjacent tissues. An upper acceptable limit is $1{ }^{\circ} \mathrm{C}$, but in cases of devices adjacent to heat-sensitive tissue, such as the atrioventricular node, lower temperature limits may be necessary. This information is then communicated to the person and to management in clear, understandable terms. In the event of a job transfer or the introduction of new technology, a reassessment should be considered. An example is shown in the appendix.

\section{Electromagnetic interference}

Devices such as cardiac pacemakers and cochlear implants may be heated by RFR, and also electromagnetic interference to their operation may occur.

Cardiac pacemakers. Historically interference with a pacemaker was a common problem, but today shield- 
ing largely prevents it (4). However, if the interfering signal is of sufficient intensity or is detected by the electrodes of the pacemaker, which act as antennas, it is possible for the timing of the pacemaker to be disturbed and an irregular or unwanted output produced. Strong electric fields $(2.5 \mathrm{kV} / \mathrm{m})$ of extremely low frequency $(50 \mathrm{~Hz})$ and RFR have been reported to cause interference $(10,11)$. Powerful RFR fields which may be encountered in the workplace should be suspect for their ability to interfere with the pulse generator (4, 11 ), although programmable pacemakers are claimed to have security systems which would prevent their reprogramming by extraneous RFR. Sources of RFR from which interference has been reported at work include electric arc welding equipment, transmitting towers and antennas, dielectric and induction heaters, and diathermy devices (11).

The following approach to pacemakers has been adopted in Telecom Australia. Details on the pacemaker are obtained, including information on its shielding, past experiences, and whether or not it reverts to a fail-safe fixed mode to avoid syncope. This information may be obtained from the cardiac center responsible for implanting the pacemaker and from the manufacturer. Details on the likely RFR exposures, including frequency, strength, modulations, and duration, are obtained, and the likelihood of adverse interaction is estimated in consultation with the manufacturer and cardiologist. The consequences of reverting to a standby rate need to be assessed in light of the job. For example, reversion to a fixed rate is potentially more serious for work at heights than work at ground level. If the risk of adverse consequences appears unlikely to lead to harm, the individual may be provided with a self-contained portable electrocardiograph, such as a Holter monitor and recorder, and monitored while working under exposed circumstances for evidence of interference to confirm the correctness of the estimation. On the basis of this result, clear advice is given to the individual and management. In the event of job transfer or new technology reassessment should be considered.

It should be noted that protective suits, similar to that used by Butrous et al (10) to protect electricity workers with pacemakers, are not suitable for RFR workers because of the inability of the suits to shield the wearer effectively from RFR fields (12).

New generation pacemakers, which sense the onset of physiological changes that occur with exercise, are particularly likely to be used in people of working age (13). Some of these devices utilize piezoelectric crystals or other sensors which may interact with RFR and lead to interference. This issue needs to be addressed during the development of these new pacemakers.

Cochlear implants. Cochlear implants are likely to be fitted to young persons who will enter the work force. Loeb (14) has described various types of cochlear im- plants. The simplest have a single channel, but the most useful, the Melbourne-Nucleus cochlear implant, has multiple channels. There are reports of the simple devices used in America receiving radio signals (15), but the Melbourne-Nucleus device has internal checking circuits which help exclude such interference (5). However, power and data are transmitted to the Melbourne-Nucleus cochlear implant by electromagnetic induction using a sequence of pulse bursts at $2.5 \mathrm{MHz}$. This frequency is also allocated for standard frequency and time transmissions in Australia and so may result in interference in special groups of RFR workers. Generally the risk of interference appears small, but RFR workers receiving implants should be advised of the risk of interference and recommended to report untoward "voices," which can be distressingly misinterpreted as hallucinations (15).

It is also recognized that pulsed RFR (radar) may cause heating of the cochlear fluid and therefore cause a clicking sound to be heard (16). This possibility should be allowed for when complaints of abnormal sounds are assessed.

\section{Discussion}

The principles of managing the adverse interaction between RFR and medical devices may be summarized as follows. First, occupational physicians or radiation protection officers and recipients of implants must be alert to the fact that adverse interactions may occur. Second, a thoughtful assessment of the frequencies and field strengths to which the worker may be exposed should take place. Third, the possible interactions should be carefully assessed through the use of mathematical modeling and laboratory tests, or a work trial should be performed. Fourth, clear advice on present or possible future risks should be given to the worker and management.

Our schemes of management are based on the assumption of safe work practices being observed such that there is compliance with the Australian standard (figure 1). It is particularly important that work practices avoid RFR burns and shocks because, in addition to the burns or shocks, the functioning of a device may be adversely affected. Parts of the aforementioned schemes of management are complex. Our experience indicates that it is prudent to use the combined skills of a scientist and a physician.

To our knowledge other national and international standards have not addressed the protection of RFR workers with implanted medical devices. It would seem timely for such standards to be developed. In light of our experience, we consider it appropriate to assess cases individually rather than create a lower, acrossthe-board RFR safety standard for workers. The numbers of staff involved are small and thus permit individual consideration. This approach also avoids 
problems of unfair discrimination in employment by being very specific about any restrictions.

Although the preceding protocols have been designed for the workplace, they may also have application in clinical settings for patients with metallic implants who are to be exposed to RFR.

\section{Acknowledgment}

This paper was published by the kind permission of Telecom Australia. Our thanks to Ms S Hayes for the preparation of the manuscript and Mr V Lubinas for the calculation of the temperature rises.

\section{References}

1. Standards Australia. Radio-frequency radiation part 1 - maximum exposure levels $-300 \mathrm{kHz}$ to $300 \mathrm{GHz}$. Sydney: Standards Australia, 1988. (Australian standard 2772-1985).

2. Lin JC. Computer methods for field intensity predictions. In: Polk CO, Postow E, ed. Handbook of biological effects of electromagnetic fields; chapter 2. Boca Raton, FL: CRC Press, 1986.

3. World Health Organization. Radio frequency and microwaves. Geneva: World Health Organization, 1981. (Environmental health criteria; 16.$)$

4. Mond H. The cardiac pacemaker. New York, NY: Grune and Straton, 1983;366-70.

5. Seligman P, Patrick J, Tong Y, Clark G, Dowell R, Crosby P. A signal processor for a multiple-electrode hearing prosthesis. Acta Otolaryngol Suppl 1984;411:
$135-9$.

6. Fleming AHJ, Joyner KH. Moment method analysis of radiation and scattering by thin wires in an infinite dissipative medium. Appl Comput Electromagn Soc J 1989; special issue: $51-74$.

7. Joyner KH, Hocking B, Fleming AHJ, Macfarlane IP. Metallic implants and exposure to radio frequency radiation. In: International Radiation Protection Association. Radiation protection in practice, proceedings of seventh international congress of the international radiation protection association. Sydney: International Radiation Protection Association, 1988:477-80.

8. Murray KB. Hazard of microwave ovens to transdermal drug delivery systems. New Engl J Med 1984;310: 721.

9. Point Study Group. One year trial of a remote-controlled implantable insulin infusion system in type 1 diabetic patients. Lancet 1988;2:866-9.

10. Butrous G, Bexton R, Barton D, Male J, Camm A. Interference with the pacemakers of two workers at electricity substations. Br J Ind Med 1983;40:462-5.

11. Canadian Centre for Occupational Health \& Safety. Possible health hazards for cardiac pacemaker wearers from exposure to electromagnetic fields. Hamilton (Ontario): Canadian Centre for Occupational Health \& Safety, 1988. (P88-5E.)

12. Joyner KH, Copeland PR, Macfarlane IP. An evaluation of a radio-frequency protective suit and electrically conductive fabrics. IEEE Trans Electromagn Compat 1989;EMC-31:129-37.

13. Anonymous. Pacemakers to make the heart beat faster with exercise [Editorial]. Lancet 1988;2:23.

14. Loeb $\mathrm{G}$. The functional replacement of the ear. Sci Am 1985;252(2):86-92.

15. Hepfner S, Skelly M. Radio-frequency interference in cochlear implants. New Engl J Med 1985;311:387.

16. Foster K, Guy A. The microwave problem. Sci Am 1986; 255:32-9.

\section{Appendix}

The assessment of the heating of an implant can be illustrated by the following case of a 32-year-old male radio technician who had undergone cardiac surgery for an ectopic focus and still had wire sutures securing his sternum.

The chest radiograh and clinical notes revealed 1-mm stainless steel wires forming loops and near-vertical twists of wire some $2 \mathrm{~mm}$ in diameter (figure A-1). These structures were classed as both rods (the wire twists) and rings for purposes of RFR interaction calculation.

The subject was predominantly exposed to frequencies between 6 and $22 \mathrm{MHz}$ at low levels $(\leq 2 \mathrm{~mW} /$ $\mathrm{cm}^{2}$ ), but the possibility existed of exposure to the allowable limit of $25 \mathrm{~mW} / \mathrm{cm}^{2}$ for up to $8 \mathrm{~h}$ with provision for a maximum short-term $(6 \mathrm{~min})$ exposure limit of up to $100 \mathrm{~mW} / \mathrm{cm}^{2}$. (See figure 1 in the introduction.) In the future he may be required to work at frequencies above $30 \mathrm{MHz}$, for which the exposure limit is $1 \mathrm{~mW} / \mathrm{cm}^{2}$ for up to $8 \mathrm{~h}$ with provision for a maximum short-term exposure limit of $5 \mathrm{~mW} / \mathrm{cm}^{2}$.

The interaction between the incident fields and the implants was assessed with the use of two methods, and the subsequent tissue temperatures were calculated. With the first method the implant was treated as a resonant structure. A perturbational analysis involving some simplifying assumptions was used to determine the ability of the sutures to heat the surrounding tissue. Because of to the high frequency at which the sutures resonate, around $1650 \mathrm{MHz}$, a planar model of the local geometry, including the sternum and surrounding tissue, was used in estimating the fields in tissue due to an external electromagnetic wave without the implant being present. Two thin wire antenna programs $(1,2)$ were then used to investigate the resulting near fields surrounding each suture. The technique of these programs is to segment the physical wire structure into small filamentary elements and apply the method of moments (3). 
This approach allowed the sutures to be modeled as combinations of rods and loops and also allowed for the investigation of the behavior of the rods or loops as individual components. Both programs required modification to enable modeling in conductive media such as biological tissue. The computed currents and near fields for the rod structures were verified (1) against data obtained by King \& Harrison (4), who derived analytic expressions for these parameters for a range of linear antennas in conductive media. The numerical and analytical approaches gave similar predictions for the electric fields at distances of half a wire radius from the tip of the rods. We tested various orientations of the model sutures to establish the orientation and location for maximum enhancement of the incident electric fields in tissue. As expected, it was found that the electric fields adjacent to the tips of the rods were maximal. The enhancement of the electric fields near the tips depends in part upon the end shape of the rods; the sharper the end, the larger the fields. Hence the fields may be singular; the calculations assumed sharp conical-shaped tips with a half angle of $45^{\circ}$.

The electric field incident on the implant due to a person being exposed to the allowable RFR limits was calculated according to the assumption of planar tissue geometry and with the methodology and electrical tissue properties given in reference 5 . The in-situ electric fields which give rise to tissue heating were calculated from the product of the incident electric field and the calculated enhancement factors around the tip of the implants. The interaction between the loops and incident vertical magnetic fields was also investigated. However, the resultant heating in adjacent tissues was significantly lower than the heating due to the concentration of the electric fields at the tip of the rods.

The heat transfer was analyzed numerically with a 3-D steady-state finite element model (6) based on the bioheat equation (5). The physical boundaries of the model extended from the outer surface of the skin through to the inner surface of the sternum, a total depth of $15.75 \mathrm{~mm}$. The width and height of the model volume were 10.35 and $13.75 \mathrm{~mm}$, respectively. The upper and lower surfaces of the volume were assumed to be insulated, and the inner surface of the sternum was clamped at $36.5^{\circ} \mathrm{C}$. Heat was conducted through the sternum and layers of skin to the skin surface according to the methodology of Saxena \& Pal (7).

The temperature rises are listed in table A-1 for the resonant range of the implants of 1650 to $3000 \mathrm{MHz}$, corresponding to wire twist lengths of 12 and $7 \mathrm{~mm}$, respectively. At the maximum short-term exposure limit (see figure 1 in the introduction) the rise in tissue temperature exceeded $1^{\circ} \mathrm{C}$.

The second method used to determine the interaction between the incident electric fields and the implants made use of a quasi-static approximation for the implant. The quasi-static approximation is valid when the dimensions of the implant are small when compared with the wavelength of RFR in tissue. The wire twists were modeled as prolate spheroids (a body obtained by rotating an ellipse about its major axis), and the enhancement in the electric field at the tip of the implant was calculated according to the work of Guy (8). The fraction of the incident electric field reaching the implant was determined from calculations of the internal electric field distribution in whole-body block models of a human (9). The in-situ electric fields giving rise to the tissue heating and the subsequent temperature rises were calculated as for the method using the resonance model of implants.

Note that the maximum for the induced electric field occurs at $80 \mathrm{MHz}$, where the whole body is acting as a resonant object. The resultant temperature rises at frequencies of $3,9.5$, and $80 \mathrm{MHz}$ are also listed in table A-1. The frequencies of 3 and $9.5 \mathrm{MHz}$ were chosen for calculation because the exposure limits reach maximum values of 100 and $10 \mathrm{~mW} / \mathrm{cm}^{2}$, respectively. (See figure 1 in the introduction).
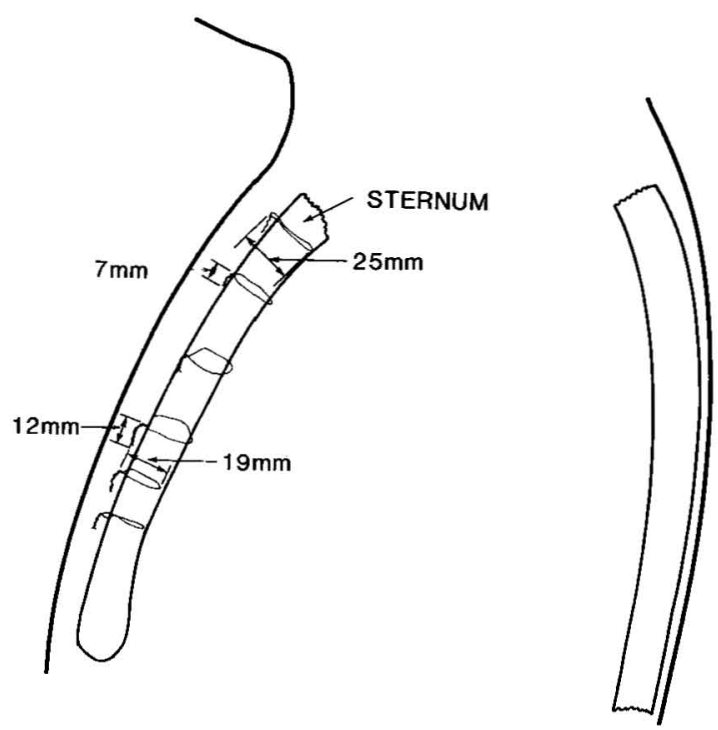

Figure A-1. Schematic drawing of a lateral chest radiograph showing wire sutures in the sternum.

Table A-1. Tissue temperature rises at the exposure limits.

\begin{tabular}{lcc}
\hline $\begin{array}{l}\text { Frequency } \\
(\mathrm{MHz})\end{array}$ & $\begin{array}{c}\text { Exposure limits } \\
\left(\mathrm{mW} / \mathrm{cm}^{2}\right)\end{array}$ & $\begin{array}{c}\text { Temperature rise } \\
\left({ }^{\circ} \mathrm{C}\right)\end{array}$ \\
\hline $1650-3000$ & 1 & 0.48 \\
$1650-3000$ & $5^{\mathrm{a}}$ & 2.4 \\
80 & 1 & 0.28 \\
80 & $5^{\mathrm{a}}$ & 1.3 \\
9.5 & 10 & 0.03 \\
3 & 100 & 0.01 \\
\hline
\end{tabular}

a Short-term exposure limits.

b At certain frequencies the tissue temperature rise approaches or exceeds $1^{\circ} \mathrm{C}$ 
After the interaction between the incident fields and the implants was assessed, the extent of heating with regard to adjacent tissue was determined. The wires were embedded in cartilaginous bone, which is relatively insensitive to heat, and in contact with skin, which is capable of readily dissipating heat. Although some of the wire loops protruded inside the rib cage, the most critical parts were found to be the tips of the wire twists, which were external to the rib cage and not in contact with heat-sensitive tissue.

The advice given to the subject and management was that work can proceed at exposure levels up to the occupational limits for frequencies below $30 \mathrm{MHz}$. However, no work at the maximum short-term exposure limits was permitted at frequencies between 50 and $150 \mathrm{MHz}$ and in the gigahertz range.

\section{References}

1. Fleming AHJ, Joyner KH. Moment method analysis of radiation and scattering by thin wires in an infinite dissipative medium. Appl Comput Electromagn Soc J
1989; special issue: $51-74$.

2. Julian A, Logan J, Rockway J. MININEC: a mininumerical electromagnetics code. San Diego, CA: US Naval Ocean Systems Centre. 1982. (Technical Document 516.)

3. Harington R. Field computation by moment methods. New York, NY: Macmillan, 1968:62-106.

4. King R, Harrison C. Antennas and waves: a modern approach. Cambridge, MA: MIT Press, 1969:137-304.

5. National Council on Radiation Protection \& Measurements. Radio frequency electromagnetic fields. Washington, DC: National Council on Radiation Protection \& Measurements, 1981. (Report; no 67.)

6. MacNeal Schendler Corporation. Heat transfer analysis, MCS.CAL, users manual. Los Angeles, CA: MacNeal Schendler Corporation, 1987. (Version 20.)

7. Saxena VP, Pal DS. Heat migration through human skin and underlying tissue under perspiration and convection - radiation conditions. Indian J Technol 1982;22:16-9.

8. Guy A. Biophysics - energy absorption and distribution London: Technical Editing and Reproduction Limited, Harford House, 1975. (AGARD lecture series no 78 on radiation hazards; report no AGARD-LS-78.)

9. Chen K, Guru B. Internal EM field and absorbed power density on human torsos induced by $1-5004 \mathrm{MHz}$ EM waves. IEEE Trans Microwave Theory Tech 1977; MTT-25:746-56. 